\title{
CONSTRUCTIONS WITH ANALYTIC SEMIGROUPS AND ABSTRACT EXPONENTIAL DECAY RESULTS FOR EIGENFUNCTIONS
}

\author{
SIGURD ANGENENT
}

\section{Presented on the occasion of H.Amann's 60th birthday}

\section{Contents}

1. Introduction

2. Resolutions

3. Localization

4. An Example

5. Exponential decay of the resolvent

6. Exponential decay of eigenfunctions

7. Exponential decay in the example

8. Application to "Fredholm solutions" of Nonlinear Elliptic Systems

\section{INTRODUCTION}

In this note we present some constructions with generators of analytic semigroups which are an abstract version of the familiar method of "freezing the coefficients" to prove elliptic estimates for differential operators with continuous coefficients or Hölder-continuous coefficients. As a side result we obtain an abstract exponential decay result for, say eigenfunctions corresponding to isolated eigenvalues.

After recalling some basic definitions in section 1.1 we first introduce a resolution of an operator $A: E_{1} \rightarrow E_{0}$ in a Banach couple $\left(E_{1}, E_{0}\right)$ (section 2 ) and then discuss localized resolutions, which allow us to formulate an abstract version of the method of "freezing the coefficients" (section 3). In section 4 we show how all this can be applied to show that systems of parabolic operators $A=\sum_{|j| \leq m} a_{j}(x) D^{j}$ generate analytic semigroups in $L^{p}$ spaces. In this example the result is certainly not new, but it illustrates the theory and suggests generalizations. In section 5 we show that the matrix elements of the resolvent of an $A \in H o l(E)$ with respect to a localized resolution are exponentially decaying, provided certain commutators are sufficiently small (In the example of systems of differential operators on $\mathbb{R}^{d}$ this would imply that the integral kernel of the resolvent is exponentially decaying.) This result immediately implies exponential localization of generalized eigenvalues corresponding to isolated eigenvalues $\lambda_{0}$ of finite multiplicity again, assuming certain commutators are small enough (see section 6). For the example it turns out one can always choose a localized resolution for which the relevant commutators are adequately small. This way we find that generalized eigenfunctions (corresponding to isolated eigenvalues with finite multiplicity) of parabolic operators $A=\sum_{|j| \leq m} a_{j}(x) D^{j}$ always decay exponentially at $x=\infty$. This fact has an implication for classical solutions of nonlinear elliptic systems, namely, if an entire solution $u(x)$ of an elliptic system of 
PDEs $F\left(u, D u, \ldots, D^{m} u\right)=0$ is such that the linearized equation at $u$ has $\lambda=0$ as isolated eigenvalue of finite multiplicity, then $\lim _{|x| \rightarrow \infty} u(x)$ exists (section 8 ).

I have found the theory of analytic semigroups particularly useful to prove short time existence and regularity results for an ever increasing class of nonlinear diffusion equations. In the middle 80ies I found the works [1][2][3] particlarly inspiring. The reader can find much information in [4], (see also [13][5][11][14][12]). There is some overlap between the theory presented here and the work in volumes I and II of [4]. I hope the reader will find the different perspective useful.

1.1. Some definitions and facts. A Banach couple $E=\left(E_{1}, E_{0}\right)$ is a pair of Banach spaces $E_{1} \subset E_{0}$ with $E_{1}$ densely embedded in $E_{0}$. An operator $A \in \mathfrak{L}\left(E_{1}, E_{0}\right)$ generates an analytic semigroup if for some $\omega>0$ all $\lambda-A: E_{1} \rightarrow E_{0}$ with $\Re \lambda>\omega$ are invertible while $\sup _{\Re \lambda>\omega}\left\|(\lambda-A)^{-1}\right\|_{\mathfrak{L}\left(E_{0}, E_{1}\right)}<\infty$.

We denote the set of $A \in \mathfrak{L}\left(E_{1}, E_{0}\right)$ which generate an analytic semigroup by $H o l(E)$. This set is open in $\mathfrak{L}\left(E_{1}, E_{0}\right)$, meaning a small perturbation (in the $E_{1} \rightarrow E_{0}$ operator norm) of a generator of an analytic semigroup also generates one. Other perturbations which do not destroy the property of generating an analytic semigroup are addition of a compact operator $K: E_{1} \rightarrow E_{0}$, or an operator $L: E_{1} \rightarrow E_{0}$ of zero relative bound (meaning for all $\varepsilon>0$ there is an $C_{\varepsilon}<\infty$ such that $\|L x\|_{E_{0}} \leq \varepsilon\|x\|_{E_{1}}+C_{\varepsilon}\|x\|_{E_{0}}$ for any $x \in E_{1}$.)

\section{Resolutions}

Consider a Banach couple $E$. By definition a resolution $E \stackrel{\delta}{\longrightarrow} F \stackrel{\varepsilon}{\longrightarrow} E$ of $E$ consists of another Banach couple $F$ and two maps of Banach couples $\delta: E \rightarrow F$ and $\varepsilon: F \rightarrow E$ which satisfy

$$
\varepsilon \delta=i d_{E}
$$

A resolution of an operator $A \in \mathfrak{L}\left(E_{1}, E_{0}\right)$ is, by definition, an operator $A^{\prime} \in \mathfrak{L}\left(F_{1}, F_{0}\right)$ where $E \stackrel{\delta}{\longrightarrow} F \stackrel{\varepsilon}{\longrightarrow} E$ is a resolution of the Banach couple $E$.

For any resolution $A^{\prime}$ of $A$ we define the commutators

$$
\begin{aligned}
{[A, \epsilon] } & =A \epsilon-\epsilon A^{\prime} \\
{[A, \delta] } & =A^{\prime} \delta-\delta A .
\end{aligned}
$$

These are bounded operators: $[A, \epsilon] \in \mathfrak{L}\left(F_{1}, E_{0}\right)$ and $[A, \delta] \in \mathfrak{L}\left(E_{1}, F_{0}\right)$. In general a resolution will be quite useless, unless the corresponding commutators are in some sense small.

We will call the resolution $A^{\prime}$ exact if the commutators $[A, \epsilon]$ and $[A, \delta]$ vanish. If these commutators are compact operators (from $F_{1}$ to $E_{0}$, or $E_{1}$ to $F_{0}$ ) then we will call the resolution compact. If the commutators are operators with relative bound zero, then we'll say the resolution has relative bound zero.

Theorem 2.1. Let $A \in \mathfrak{L}\left(E_{1}, E_{0}\right)$ have a resolution $A^{\prime} \in \mathfrak{L}\left(F_{1}, F_{0}\right)$, and assume that this resolution is either compact, or of relative bound zero. Then $A^{\prime} \in \operatorname{Hol}(F)$ implies $A \in H o l(E)$. 
Proof. Let $A^{\prime} \in \mathfrak{L}\left(F_{1}, F_{0}\right)$ be the given resolution and assume that the resolution is compact. It follows from $\epsilon \delta=i d_{E}$ that

$$
\pi_{F}=\delta \epsilon
$$

is a bounded projection in $F$. This projection allows us to split $F$ as

$$
F=\operatorname{range}(\delta) \oplus \operatorname{kern}(\epsilon) .
$$

The matrix of the linear operator $A^{\prime}$ with respect to this splitting can be written as

$$
A^{\prime}=\left(\begin{array}{cc}
P & Q \\
R & S
\end{array}\right)
$$

with $P \in \mathfrak{L}\left(\operatorname{range}(\delta)_{1}\right.$, range $\left.(\delta)_{0}\right), R \in \mathfrak{L}\left(\operatorname{range}(\delta)_{1}, \operatorname{kern}(\epsilon)_{0}\right)$, etc.

The off diagonal parts of $A^{\prime}, R$ and $Q$, are given by

$$
\begin{aligned}
& R=\left(1-\pi_{F}\right) A^{\prime} \pi_{F}=\left[A^{\prime}, \pi_{F}\right] \pi_{F} \\
& Q=\pi_{F} A^{\prime}\left(1-\pi_{F}\right)=\pi_{F}\left[\pi_{F}, A^{\prime}\right]
\end{aligned}
$$

and since

$$
\left[A^{\prime}, \pi_{F}\right]=\left[A^{\prime}, \delta \epsilon\right]=[A, \delta] \epsilon+\delta[A, \epsilon]
$$

the commutator $\left[A^{\prime}, \pi_{F}\right]$ is a compact operator. Therefore $R$ and $Q$ are also compact. Compact perturbations of an $A^{\prime} \in \operatorname{Hol}(F)$ still belong to $\operatorname{Hol}(F)$, so that

$$
\left(\begin{array}{cc}
P & 0 \\
0 & S
\end{array}\right) \in \operatorname{Hol}(F),
$$

which implies that $P \in \operatorname{Hol}(\operatorname{range}(\delta))$. But $\delta$ is an isomorphism onto its range, with inverse $\epsilon$, so

$$
\epsilon A^{\prime} \delta=\epsilon P \delta \in \operatorname{Hol}(E) .
$$

Finally,

$$
\epsilon A^{\prime} \delta-A=\epsilon A^{\prime} \delta-\epsilon \delta A=\epsilon[A, \delta]
$$

is again a compact operator and we may conclude that $A \in \operatorname{Hol}(E)$.

If the resolution is not compact but of vanishing relative bound instead, then the same proof will work. Q.E.D.

Given a resolution of $A \in \mathfrak{L}\left(E_{1}, E_{0}\right)$ one can try to express the resolvent of $A$ in terms of the resolvent of $A^{\prime}$. Let

$$
R^{\prime}(\lambda)=\left(\lambda-A^{\prime}\right)^{-1}
$$

be the resolvent of $A^{\prime}$, for some $\lambda \in \mathbb{C}$.

As a first approximation to the resolvent of $A$ we introduce

$$
S(\lambda)=\epsilon R^{\prime}(\lambda) \delta .
$$

To measure by how much $S(\lambda)$ fails to be the resolvent of $A$, we consider

$$
T(\lambda)=1_{E}-(\lambda-A) S(\lambda)=[A, \epsilon] R^{\prime}(\lambda) \delta .
$$


Formally a right inverse for $\lambda-A$ is given by

$$
\begin{aligned}
& \text { right- }(\lambda-A)^{-1}=S(\lambda)[(\lambda-A) S(\lambda)]^{-1} \\
& \quad=S(\lambda)\left[1_{E}-T(\lambda)\right]^{-1} \\
& =S(\lambda)\left[1_{E}+T(\lambda)+T(\lambda)^{2}+\cdots\right] \\
& =\sum_{k=1}^{\infty} \epsilon \circ R^{\prime}(\lambda)\left\{\delta[A, \epsilon] R^{\prime}(\lambda)\right\}^{k} \delta \\
& =\epsilon R^{\prime}(\lambda)\left[\sum_{k=1}^{\infty}\left\{\delta[A, \epsilon] R^{\prime}(\lambda)\right\}^{k}\right] \delta .
\end{aligned}
$$

If the infinite series converges in the operator norm then the terms of the series decay at least exponentially, and the series represents an actual right inverse for $\lambda-A$. Thus the series converges if and only if for some $k \geq 1$ one has

$$
\left\|\left\{\delta[A, \epsilon] R^{\prime}(\lambda)\right\}^{k}\right\|_{\mathfrak{L}\left(F_{0}\right)}<1
$$

or, what is equivalent, if the spectral radius of $\delta[A, \epsilon] R^{\prime}(\lambda) \in \mathfrak{L}\left(E_{0}\right)$ is less than one.

To obtain a left inverse for $\lambda-A$ one considers

$$
\hat{T}(\lambda)=1_{E}-S(\lambda)(\lambda-A)=-\epsilon R^{\prime}(\lambda)[A, \delta],
$$

and one tries to sum the series

$$
\begin{aligned}
\text { left- } & (\lambda-A)^{-1}= \\
\quad= & {\left[1_{E}+\hat{T}(\lambda)+\hat{T}(\lambda)^{2}+\cdots\right] S(\lambda) } \\
\quad= & \epsilon\left[\sum_{k=1}^{\infty}\left\{-R^{\prime}(\lambda)[A, \delta] \epsilon\right\}^{k}\right] R^{\prime}(\lambda) \delta .
\end{aligned}
$$

Again, a necessary and sufficient condition for summability of the series is that the spectrum of $R^{\prime}(\lambda)[A, \delta] \epsilon$ as operator on $F_{0}$ is contained in the open unit disk. We can summarize this as follows.

Theorem 2.2. If $A \in \mathfrak{L}\left(E_{1}, E_{0}\right)$ has a resolution $A^{\prime}$, and $\lambda \in \mathbb{C}$ lies in the resolvent set of $A^{\prime}$, then $\lambda$ also belongs to the resolvent set of $A$ if

$$
r_{\text {spec }}\left(R^{\prime}(\lambda)[A, \delta] \epsilon\right)<1 \quad \text { and } r_{\text {spec }}\left(\delta[A, \epsilon] R^{\prime}(\lambda)\right)<1 .
$$

The resolvent of $A$ at $\lambda$ is then given by

$$
\begin{aligned}
(\lambda-A)^{-1} & =\epsilon R^{\prime}(\lambda)\left[\sum_{k=0}^{\infty}\left\{\delta[A, \epsilon] R^{\prime}(\lambda)\right\}^{k}\right] \delta \\
& =\epsilon\left[\sum_{k=0}^{\infty}\left\{-R^{\prime}(\lambda)[A, \delta] \epsilon\right\}^{k}\right] R^{\prime}(\lambda) \delta
\end{aligned}
$$

\section{LOCALIZATION}

Let $E \stackrel{\delta}{\longrightarrow} F \stackrel{\epsilon}{\longrightarrow} E$ be a resolution of the Banach couple $E$. We will say that the resolution is localized if the Banach couple $F$ can be written as a direct sum

$$
F=\ell_{p}-\bigoplus_{\alpha \in \mathfrak{A}} F^{(\alpha)}
$$


where $\mathfrak{A}$ is a finite or countable index set. More precisely, for each $\alpha \in \mathfrak{A}$ we have a Banach couple $F^{(\alpha)}$ for which $F_{j}^{(\alpha)}$ is a closed subspace of $F_{j}(j=0,1)$, and each $x \in F_{j}$ can be written in exactly one way as a norm convergent sum

$$
x=\sum_{\alpha \in \mathfrak{A}} x^{(\alpha)}
$$

and the $F_{j}$ norm of $x$ is equivalent to the expression

$$
\left(\sum_{\alpha \in \mathfrak{A}}\left\|x^{(\alpha)}\right\|_{F_{j}}^{p}\right)^{1 / p} .
$$

Throughout this article all direct sums $\bigoplus_{\alpha \in \mathfrak{A}}$ will be understood to be $\ell_{p}$ sums in the sense just explained.

We will say that an $x \in F_{i}$ has finite support if $x \in F_{i}^{\left(\alpha_{1}\right)} \bigoplus \ldots \oplus F_{i}^{\left(\alpha_{m}\right)}$ for a finite number of $\alpha_{1}, \ldots, \alpha_{m} \in \mathfrak{A}$. We denote the space of $x$ with finite support by $F_{i}^{\text {fin }}$. Since every $x \in F_{i}$ is a norm convergent sum of $x^{(\alpha)}$ 's the space $F_{i}^{\text {fin }}$ is dense in $F_{i}$.

Given such a direct sum, we define the canonical projections $p_{\alpha}: F \rightarrow F^{(\alpha)}$ and inclusions $i_{\alpha}: F^{(\alpha)} \rightarrow F$ by

$$
\left\{\begin{array}{c}
p_{\alpha}(x)=x^{(\alpha)} \text { if } x=\sum_{\alpha \in \mathfrak{A}} x^{(\alpha)} \\
i_{\alpha}(x)=x \text { for all } x \in F_{j}^{(\alpha)}(j=0,1)
\end{array}\right.
$$

If $T \in \mathfrak{L}\left(F_{i}, F_{j}\right)(i, j=0,1)$ is a bounded operator, then we can consider its associated matrix $\left\{T_{\alpha \beta}\right\}$, with $T_{\alpha \beta} \in \mathfrak{L}\left(F_{i}^{(\beta)}, F_{j}^{(\alpha)}\right)$, defined by

$$
T_{\alpha \beta}=p_{\alpha} T i_{\beta} .
$$

For operators $T: E \rightarrow E$ we will also define the "matrix elements" $T_{\alpha \beta} \in \mathfrak{L}\left(F_{i}^{(\beta)}, F_{j}^{(\alpha)}\right)$ by

$$
T_{\alpha \beta}=p_{\alpha} \delta T \varepsilon i_{\beta} .
$$

The graph of a localized resolution. We can define the matrix of the projection $\pi_{F}=\delta \epsilon$

$$
\pi_{\alpha \beta}=p_{\alpha} \pi_{F} i_{\beta},
$$

and we can define a graph $\mathfrak{G}(E, F)$, whose vertices are the elements of $\mathfrak{A}$, and in which $\alpha$ and $\beta$ are connected if either $\pi_{\alpha \beta} \neq 0$ or $\pi_{\beta \alpha} \neq 0$.

We will always assume that the graph $\mathfrak{G}(E, F)$ is uniformly locally finite. This means by definition that

$$
n_{\mathfrak{G}} \stackrel{\text { def }}{=} \max _{\alpha \in \mathfrak{A}}\{\text { number of edges containing } \alpha\}
$$

is finite.

Let $A \in \mathfrak{L}\left(E_{1}, E_{0}\right)$ be given. Then an operator $A^{\prime} \in \mathfrak{L}\left(F_{1}, F_{0}\right)$ will be called a localized resolution of $A$, if

1. The decomposition $F=\bigoplus_{\alpha \in \mathfrak{A}} F^{(\alpha)}$ is $A^{\prime}$ invariant.

2. The operator $\delta A \epsilon$ is dominated by the projection $\pi_{F}$, in the sense that $(\delta A \epsilon)_{\alpha \beta}=0$ whenever $\pi_{\alpha \beta}=0$. 
By $A^{\prime}$ invariance we mean:

$$
A^{\prime}\left(F_{1}^{(\alpha)}\right) \subset F_{0}^{(\alpha)} \quad(\forall \alpha \in \mathfrak{A}) .
$$

Lemma 3.1. If $A^{\prime} \in H o l(F)$, then the restriction $A^{(\alpha)}$ of $A^{\prime}$ to $F^{(\alpha)}$ belongs to $\operatorname{Hol}\left(F^{(\alpha)}\right)$.

Proof. Let $\lambda$ belong to the resolvent set of $A^{\prime}$. Then (7) trivially implies

$$
\left(\lambda-A^{\prime}\right)^{-1}\left(F_{0}^{(\alpha)}\right) \subset F_{1}^{(\alpha)} \quad(\forall \alpha \in \mathfrak{A}) .
$$

Indeed, if $f_{\alpha} \in F_{0}^{(\alpha)}$ then we can write $g=\left(\lambda-A^{\prime}\right)^{-1} f_{\alpha}$ as $g=\sum_{\beta \in \mathfrak{A}} g_{\beta}$, with $g_{\beta} \in F_{1}^{(\beta)}$, and it follows from

$$
\left(\lambda-A^{\prime}\right) g=\sum_{\beta \in \mathfrak{A}}\left(\lambda-A^{\prime}\right) g_{\beta}=f_{\alpha} \in F_{0}^{(\alpha)}
$$

and the uniqueness of the decomposition of $g$ into the $g_{\beta}$ 's that $g_{\beta}=0$ for all $\beta \neq \alpha$. Hence $g \in F_{1}^{(\alpha)}$.

Since the norm of the resolvent restricted to $F_{0}^{(\alpha)}$ can never be more than its norm on $F_{0}$, this shows that $A^{(\alpha)} \in \operatorname{Hol}\left(F^{(\alpha)}\right)$. Q.E.D.

As a converse to this lemma we have the following observation.

Lemma 3.2. Assume that all the $A^{(\alpha)}$ 's belong to $\operatorname{Hol}\left(F^{(\alpha)}\right)$, that an $\omega \in \mathbb{R}$ exists for which any $\lambda \in \mathbb{C}$ with $\Re(\lambda) \geq \omega$ belongs to the resolvent set of all the $A^{(\alpha)}$ 's, and for which

$$
M=\sup _{\Re(\lambda) \geq \omega} \sup _{\alpha \in \mathfrak{A}}\left\|\left(\lambda-A^{(\alpha)}\right)^{-1}\right\|_{\mathfrak{L}\left(F_{0}^{(\alpha)}, F_{1}^{(\alpha)}\right)}<\infty .
$$

Then $A^{\prime} \in \operatorname{Hol}(F)$.

Indeed, if $\Re(\lambda) \geq \omega$ then $\lambda$ is in the resolvent set of $A^{\prime}$. The resolvent is given by

$$
\left(\lambda-A^{\prime}\right)^{-1}\left\{f_{\alpha}\right\}=\left\{\left(\lambda-A^{(\alpha)}\right)^{-1} f_{\alpha}\right\}
$$

and its $\mathfrak{L}\left(F_{0}, F_{1}\right)$ norm does not exceed $M$.

\section{An EXAMPLE}

Let $E=\left(W_{p}^{m}\left(\mathbb{R}^{d} ; \mathbb{R}^{n}\right), L_{p}\left(\mathbb{R}^{d} ; \mathbb{R}^{n}\right)\right)$ with $1<p<\infty$, and consider the $m^{\text {th }}$-order (matrix) differential operator

$$
A=\sum_{|j| \leq m} a_{j}(x) D^{j} \in \mathfrak{L}\left(E_{1}, E_{0}\right) .
$$

Here the coefficients $a_{j}(x)$ are continuous $d \times d$ matrix valued functions, and the subscript $j$ is a multiindex. We will show that $A \in \operatorname{Hol}(E)$, if the $a_{j}(x)$ are uniformly continuous, and if the principal symbol

$$
A_{m}(x, \xi) \stackrel{\text { def }}{=} \sum_{|j|=m} a_{j}(x)(-i \xi)^{j}
$$

satisfies

$$
\left\|\left(\lambda I-A_{m}(x, \xi)\right)^{-1}\right\| \leq \frac{c}{|\lambda|+|\xi|^{m}}
$$


for all $x \in \mathbb{R}^{d}$, all $\xi \in \mathbb{C}^{d}$, all $\lambda \in \mathbb{C}$ with $\Re \lambda \geq c$, and some constant $c>0$. This is of course well known, but the proof we give below generalizes to many other situations and should also motivate the constructions which follow in this section.

Choose a bounded open symmetric neighbourhood $O \subset \mathbb{R}^{d}$ of the origin, whose translates $\left\{O+\alpha \mid \alpha \in \mathbb{Z}^{d}\right\}$ cover $\mathbb{R}^{d}$ (e.g. a sufficiently large sphere). We then define for any "scale" $r>0$ and $\alpha \in \mathbb{Z}^{d}$ the set

$$
O_{\alpha ; r} \stackrel{\text { def }}{=}\{r(\alpha+x) \mid x \in O\}
$$

For any $r>0$ the $O_{\alpha ; r}$ cover $\mathbb{R}^{d}$. This covering is uniformly locally finite: since $O$ is bounded it contains only a finite number $N$ of lattice points, and hence any $O_{\alpha ; r}$ intersects exactly $N$ other $O_{\beta ; r} \mathrm{~s}$.

Next we construct a suitable partition of unity. Choose $0 \leq \psi \in C_{c}^{\infty}(O)$ so that the translates by $\mathbb{Z}^{d}$ of $\{x \mid \psi(x)>0\}$ also cover $\mathbb{R}^{d}$. Define

$$
\varphi_{\alpha}(x) \stackrel{\text { def }}{=} \frac{\psi(x-\alpha)}{\sqrt{\sum_{\beta \in \mathbb{Z}^{d}} \psi(x-\beta)^{2}}}
$$

and

$$
\varphi_{\alpha ; r}(x) \stackrel{\text { def }}{=} \varphi_{\alpha}\left(\frac{x}{r}\right)
$$

Then

$$
\sum_{\alpha \in \mathbb{Z}^{d}} \varphi_{\alpha ; r}(x-\alpha)^{2}=1 \quad\left(\forall x \in \mathbb{R}^{d}\right) .
$$

We now describe the resolution. The index set will be $\mathfrak{A}=\mathbb{Z}^{d}$, and for each $\alpha \in \mathbb{Z}^{d}$ we put

$$
F^{(\alpha)}=\left(W_{p}^{m}\left(\mathbb{R}^{d} ; \mathbb{R}^{n}\right), L_{p}\left(\mathbb{R}^{d} ; \mathbb{R}^{n}\right)\right)
$$

so that the Banach couple $F$ is defined by

$$
F=\ell_{p}-\bigoplus_{\alpha \in \mathfrak{A}} F^{(\alpha)}
$$

Thus $F_{j}(j=0,1)$ is the space of all sequences $\left\{f_{\alpha}\right\}_{\alpha \in \mathbb{Z}^{d}}$ of functions $f_{\alpha} \in F_{j}^{(\alpha)}$ for which the sum

$$
\left\|\left\{f_{\alpha}\right\}_{\alpha \in \mathfrak{A}}\right\|_{F_{j}}^{p}=\sum_{\alpha \in \mathfrak{A}}\left\|f_{\alpha}\right\|_{F_{j}^{(\alpha)}}^{p}
$$

is finite.

We construct the morphisms $E \stackrel{\delta}{\rightarrow} F \stackrel{\epsilon}{\rightarrow} E$ using the partition of unity $\left\{\varphi_{\alpha}\right\}$. For any $f \in E_{j}(j=0,1)$ we define the $\alpha^{\text {th }}$ component of $\delta f$ to be

$$
(\delta f)_{\alpha}=\varphi_{\alpha ; r}(x) f(x),
$$

and for any $\left\{f_{\alpha}\right\}_{\alpha \in \mathfrak{A}} \in F_{j}(j=0,1)$ we put

$$
\left(\epsilon\left\{f_{\alpha}\right\}\right)(x)=\sum_{\beta \in \mathfrak{A}} \varphi_{\beta ; r}(x) f_{\beta}(x) .
$$


Observe that this last sum is locally finite and that both $\epsilon: F_{j}^{(\alpha)} \rightarrow E_{j}$ and $\delta: E_{j} \rightarrow F_{j}^{(\alpha)}$ are bounded operators. As an example, let us verify that $\delta: F_{1}^{(\alpha)} \rightarrow E_{1}$ is bounded. Let $f \in W_{p}^{m}\left(\mathbb{R}^{d} ; \mathbb{R}^{n}\right)$ be given. Then

$$
\begin{aligned}
\|\delta f\|_{F_{1}}^{p} & =\sum_{\alpha \in \mathfrak{A}}\left\|(\delta f)_{\alpha}\right\|_{F_{1}^{(\alpha)}}^{p} \\
& =\sum_{\alpha \in \mathfrak{A}}\left\|\varphi_{\alpha} f\right\|_{W_{p}^{m}\left(\mathbb{R}^{d} ; \mathbb{R}^{n}\right)}^{p} \\
& \leq c \sum_{\alpha \in \mathfrak{A}}\|f\|_{W_{p}^{m}\left(O_{\alpha ; r}\right)}^{p} \\
& \leq N c\|f\|_{E_{1}} .
\end{aligned}
$$

where, as above, $N$ is the number of lattice points in $O$. So $\delta: E_{1} \rightarrow F_{1}$ is indeed bounded.

For any $f \in F_{j}$ one has

$$
(\epsilon \delta f)(x)=\sum_{\beta \in \mathfrak{A}} \varphi_{\beta}(x)^{2} f(x)=f(x)
$$

so that $\epsilon \delta=1_{E}$, and therefore $E \stackrel{\delta}{\longrightarrow} F \stackrel{\epsilon}{\longrightarrow} E$ is indeed a localized resolution of $E$.

The projection $\pi_{F}=\delta \epsilon$ acts as follows:

$$
\left(\pi_{F}\left\{f_{\alpha}\right\}\right)_{\alpha}(x)=\sum_{\beta \in \mathfrak{A}} \varphi_{\alpha}(x) \varphi_{\beta}(x) f_{\beta}(x),
$$

from which one can see what the graph $\mathfrak{G}(E, F)$ is: the vertices are the lattice points $\alpha \in \mathfrak{A}$, and $\alpha, \beta \in \mathfrak{A}$ are connected if and only if $\alpha-\beta \in O$. In particular this graph is uniformly locally finite, and we have $n_{\mathfrak{G}}=N$.

Interpretation of the matrix coefficients $T_{\alpha \beta}=p_{\alpha} \delta T \varepsilon i_{\beta}$ of an operator $T: E_{1} \rightarrow$ $E_{0}$. It is evident that for any $f \in E_{1}$ one has

$$
T_{\alpha \beta}(f)=\varphi_{\alpha ; r} T\left(\varphi_{\beta ; r} f\right)
$$

so that $T_{\alpha \beta}(f)$ more or less represents the component in $O_{\alpha ; r}$ of the image under $T$ of the component in $O_{\beta ; r}$ of $f$.

Next, we introduce an operator $A^{\prime} \in \mathfrak{L}\left(F_{1}, F_{0}\right)$ by saying how it acts on each $F_{1}^{(\alpha)}$ :

$$
\left(A^{(\alpha)} f_{\alpha}\right)(x)=\sum_{|j| \leq m} a_{j}^{(\alpha)}(x) D^{j} f_{\alpha}(x) \quad(\forall x \in \mathbb{R})
$$

for any $f_{\alpha} \in F_{1}^{(\alpha)}$. The coefficients $a_{j}^{(\alpha)}(x)$ coincide with the coefficients $a_{j}(x)$ of the original operator $A$ on the support of $\varphi_{\alpha}$. Outside of these supports the $a_{j}^{(\alpha)}(x)$ are continuous extensions of the $a_{j}(x)$ for which

$$
\underset{x \in \mathbb{R}^{d}}{\mathrm{Osc}} a_{j}^{(\alpha)}(x)=\underset{x \in \operatorname{spt} \varphi_{\alpha}}{\mathrm{Osc}} a_{j}^{(\alpha)}(x)
$$

holds. ${ }^{1}$

\footnotetext{
${ }^{1}$ For vector valued functions $f$ one should defined osc $f$ to be the smallest $r>0$ such that the range of $f$ is contained in a ball of radius $r / 2$.
} 
The operator $A^{\prime}$ defined by $A^{\prime}\left\{f_{\alpha}\right\}=\left\{A^{(\alpha)} f_{\alpha}\right\}$ is a resolution of $A$. Observe that the $\alpha^{\text {th }}$ component of $A^{\prime}$, i.e. $A^{(\alpha)}$, coincides with $A$ on the support of $\varphi_{\alpha}$, but is chosen arbitrarily outside of this set.

In order to see how good the resolution $A^{\prime}$ is, we will compute the commutators $[A, \epsilon]$ and $[A, \delta]$. It turns out that these commutators do not depend on what the $A^{(\alpha)}$ 's do outside of the support of $\varphi_{\alpha}$. This gives us the freedom to choose $A^{(\alpha)}$ any way we like on the complement of the support of $\varphi_{\alpha}$. The particular choice which we have made ensures that the coefficients $a_{j}^{(\alpha)}(x)$ of $A^{(\alpha)}$ do not oscillate more than the $a_{j}(x)$ do on $O_{\alpha ; r}$.

In general the commutator of a differential operator with a multiplication operator is another differential operator of lower order. The point of the following computations is to show that $[A, \epsilon]$ and $[A, \delta]$ are made up of differential operators of order $m-1$.

If $\left\{f_{\alpha}\right\} \in F_{1}$, then

$$
\begin{aligned}
{[A, \epsilon] \cdot\left\{f_{\alpha}\right\} } & =A\left\{\sum_{\alpha \in \mathfrak{A}} \varphi_{\alpha ; r} f_{\alpha}\right\}-\sum_{\alpha \in \mathfrak{A}} \varphi_{\alpha ; r} A^{(\alpha)}\left\{f_{\alpha}\right\} \\
& =\sum_{|j| \leq m} \sum_{\alpha \in \mathfrak{A}} a_{j}(x)\left[D^{j}, \varphi_{\alpha ; r}\right] f_{\alpha} \\
& =\sum_{\alpha \in \mathfrak{A}} a_{j}(x) \sum_{|j| \leq m} \sum_{0<k \leq j} \gamma_{j, k} D^{k}\left(\varphi_{\alpha ; r}\right) D^{j-k}\left(f_{\alpha}\right) \\
& =\sum_{|j| \leq m} \sum_{0<k \leq j} \sum_{\alpha \in \mathfrak{A}} b_{j ; k}^{(\alpha)}(x) \frac{D^{j-k}\left(f_{\alpha}\right)}{r^{|k|}}
\end{aligned}
$$

where the $\gamma_{j, k}$ are fixed integers which appear as coefficients when one expands the commutator $\left[D^{j}, \varphi_{\alpha ; r}\right]$, and where

$$
b_{j ; k}^{(\alpha)}(x)=a_{j}(x) \gamma_{j, k}\left(D^{k} \varphi\right)\left(\frac{x-\alpha}{r}\right) .
$$

The coefficients $b_{j ; k}^{(\alpha)}(x)$ are uniformly bounded, i.e. there is a constant $M<\infty$ such that

$$
\left|b_{j ; k}^{(\alpha)}(x)\right|<M
$$

for all $x \in \mathbb{R}^{d}$ and all multiindices $0<k \leq j$, and the support of $b_{j ; k}^{(\alpha)}(x)$ is contained in $O_{\alpha ; r}$. Consequently the operator $[A, \epsilon]$ is bounded from $\bigoplus_{\alpha \in \mathfrak{A}} W_{p}^{m-1}\left(\mathbb{R}^{d} ; \mathbb{R}^{n}\right)$ to $L_{p}\left(\mathbb{R}^{d} ; \mathbb{R}^{n}\right)$, and therefore $[A, \epsilon]$, by the interpolation inequality

$$
\|u\|_{W_{p}^{m-1}} \leq C\|u\|_{W_{p}^{m}}^{1-1 / m}\|u\|_{L_{p}}^{1 / m}
$$

is an operator of zero relative bound from $F_{1}$ to $E_{0}$. 
As for $[A, \delta]$, we compute that for $f \in E_{1}$ one has

$$
\begin{aligned}
([A, \delta] \cdot f)_{\alpha} & =A^{(\alpha)}\left(\varphi_{\alpha}(x) f(x)\right)-\varphi_{\alpha}(x) A f(x) \\
& =\sum_{|j|=m} a_{j}(x)\left[D^{j}, \varphi_{\alpha ; r}\right] f \\
& =\sum_{|j|=m} \sum_{0<k \leq j} \gamma_{j, k} D^{k}\left(\varphi_{\alpha ; r}\right) D^{j-k}(f) \\
& =\sum_{|j|=m} \sum_{0<k \leq j} b_{j ; k}^{(\alpha)}(x) \frac{D^{j-k}(f)}{r^{k}}
\end{aligned}
$$

Again the coefficients $b_{j ; k}^{(\alpha)}(x)$ are uniformly bounded, from which one deduces that $[A, \delta]$ is a bounded operator from $W_{p}^{m-1}\left(\mathbb{R}^{d} ; \mathbb{R}^{n}\right)$ to $\bigoplus_{\alpha \in \mathfrak{A}} L_{p}\left(\mathbb{R}^{d} ; \mathbb{R}^{n}\right)$. The interpolation inequality (18) implies that $[A, \delta]: E_{1} \rightarrow F_{0}$ has zero relative bound.

So $A^{\prime}$ is a resolution of $A$ with vanishing relative bound, and if we can show that $A^{\prime} \in$ $\operatorname{Hol}(F)$, then we have also shown that $A \in H o l(E)$.

In order to verify that $A^{\prime} \in \operatorname{Hol}(F)$ we consider each $A^{(\alpha)}$ separately, with the intention of using lemma (3.2). By assumption the coefficients $a_{j}^{(\alpha)}(x)$ are almost constant if one chooses the scale $r>0$ small enough. Thus, given any $\mu_{0}>0$ one can choose $r>0$ so small that constant $d \times d$ matrices $\bar{a}_{j}^{(\alpha)}$ exist for which

$$
\left|a_{j}^{(\alpha)}(x)-\bar{a}_{j}^{(\alpha)}\right| \leq \mu_{0}, \quad \forall x \in \mathbb{R}^{d}
$$

holds for all $\alpha \in \mathfrak{A}$. So the $A^{(\alpha)}$ 's are uniformly small perturbations of the constant coefficient operators

$$
A_{0}^{(\alpha)}=\sum_{|j| \leq m} \bar{a}_{j}^{(\alpha)} D^{j},
$$

all of which belong to $\operatorname{Hol}\left(F^{(\alpha)}\right)$. The resolvent of $A_{0}^{(\alpha)}$ is given by convolution with

$$
\mathfrak{F}^{-1}\left\{\left(\lambda-\sum_{|j| \leq m} \bar{a}_{j}^{(\alpha)}(-i \xi)^{j}\right)^{-1}\right\}
$$

where $\mathfrak{F}$ denotes the Fourier transform. An appropriate multiplier theorem then provides us with a constant $M<\infty$ such that

$$
\left\|\left(\lambda-A_{0}^{(\alpha)}\right)^{-1}\right\|_{\mathfrak{L}\left(F_{0}^{(\alpha)}, F_{1}^{(\alpha)}\right)} \leq M
$$

whenever $\Re(\lambda) \geq \omega$ for some fixed $\omega>0$. Therefore if the size $\mu_{0}$ of the perturbation $A^{(\alpha)}-A_{0}^{(\alpha)}$ is small enough, the $A^{(\alpha)}$ will satisfy the same estimate with $2 M$ instead of $M$. By lemma (3.2) this implies that $A^{\prime} \in \operatorname{Hol}(F)$, and thus $A \in \operatorname{Hol}(E)$.

\section{EXPONENTIAL DECAY OF THE RESOLVENT}

Consider a localized resolution $E \stackrel{\delta}{\longrightarrow} F \stackrel{\varepsilon}{\longrightarrow} E, A^{\prime} \in H o l(F)$, of $A \in H o l(E)$ and assume that for some $\lambda$ in the resolvent set of $A^{\prime}$ one has

$$
\left\|\delta[A, \epsilon] R^{\prime}(\lambda)\right\|_{L\left(F_{0}, F_{1}\right)} \leq \vartheta \quad \text { and } \quad\left\|R^{\prime}(\lambda)[\delta, A] \epsilon\right\|_{L\left(F_{0}, F_{1}\right)} \leq \vartheta
$$

for some $\vartheta<1$. By Theorem $2.2 \lambda$ belongs to the resolvent set of $A$, and the resolvent is 
given by (1) or (2). One can write (2) as

$$
(\lambda-A)^{-1}=\epsilon R^{\prime}(\lambda)\left[1+D+D^{2}+\ldots+D^{k}+\ldots\right] \delta
$$

where

$$
D=\delta[A, \epsilon] R^{\prime}(\lambda)
$$

Using this expression we will estimate the matrix elements of $(\lambda-A)^{-1}$, as defined in (5), i.e.

$$
\begin{aligned}
\left\{(\lambda-A)^{-1}\right\}_{\alpha \beta} & =p_{\alpha} \delta(\lambda-A)^{-1} \varepsilon i_{\beta} \\
& =p_{\alpha} \delta \epsilon R^{\prime}(\lambda)\left[1+D+D^{2}+\ldots+D^{k}+\ldots\right] \delta \varepsilon i_{\beta} \\
& =p_{\alpha} \pi_{F} R^{\prime}(\lambda)\left[1+D+D^{2}+\ldots+D^{k}+\ldots\right] \pi_{F} i_{\beta}
\end{aligned}
$$

To begin we consider the operator $D: F_{0} \rightarrow F_{0}$ and estimate the matrix elements of its powers $D^{k}, k=1,2, \ldots$ Recall that these matrix elements are defined as in (4), i.e. $D_{\alpha \beta}=p_{\alpha} D i_{\beta}$, etc. For the matrix elements of $D^{k}$ we then have

$$
\left(D^{k}\right)_{\alpha \beta}=\sum_{\alpha_{1}} \ldots \sum_{\alpha_{k}} D_{\alpha \alpha_{1}} D_{\alpha_{1} \alpha_{2}} \ldots D_{\alpha_{k} \beta}
$$

Lemma 5.1. The operator $D$ is dominated by the projection $\pi_{F}$, i.e. $D_{\alpha \beta} \neq 0$ only if $\alpha$ and $\beta$ are adjacent in the graph $\mathfrak{G}$.

The only nonzero terms in the sum (22) are those for which the graph $\mathfrak{G}$ contains a path of length $k+1$ from $\alpha$ to $\beta$.

Proof. The resolvent $R^{\prime}(\lambda)$ of the resolution $A^{\prime}$ is local, so we must show that $\delta[A, \epsilon]$ is dominated by the projection $\pi_{F}$. This follows from $\delta[A, \varepsilon]=\delta A \varepsilon-A^{\prime} \delta \varepsilon=\delta A \varepsilon-A^{\prime} \pi_{F}$. The first term in this expression is by assumption dominated by $\pi_{F}$, while the second is also dominated by $\pi_{F}$ since $A^{\prime}$ is local.

If $D_{\alpha \alpha_{1}} D_{\alpha_{1} \alpha_{2}} \ldots D_{\alpha_{k} \beta} \neq 0$ then $\alpha$ is adjacent to $\alpha_{1}, \alpha_{1}$ is adjacent to $\alpha_{2}$, etc, so $\left\{\alpha, \alpha_{1}, \ldots, \alpha_{k}, \beta\right\}$ is a path of length $k+1$ from $\alpha$ to $\beta$. Q.E.D.

Continuing the computation in (21) we find that the $\alpha \beta$ element of the resolvent $(\lambda-A)^{-1}$ is given by

$$
\left\{(\lambda-A)^{-1}\right\}_{\alpha \beta}=\sum \pi_{\alpha \alpha_{1}} R^{\prime}(\lambda) D_{\alpha_{1} \alpha_{2}} D_{\alpha_{2} \alpha_{3}} \ldots D_{\alpha_{k} \alpha_{k+1}} \pi_{\alpha_{k+1} \beta}
$$

where the summation runs over all paths $\alpha \rightarrow \alpha_{1} \rightarrow \ldots \rightarrow \alpha_{k+1} \rightarrow \beta$ of length $k+2$ at least 2 .

Theorem 5.2. If we assume that $\vartheta n_{\mathfrak{G}}<\frac{1}{2}$ then the operator norm from $F_{0}^{(\beta)}$ to $F_{1}^{(\alpha)}$ of $\left\{(\lambda-A)^{-1}\right\}_{\alpha \beta}$ is bounded by $C\left(\vartheta n_{\mathfrak{G}}\right)^{-2}\left\|R^{\prime}(\lambda)\right\|_{\mathfrak{L}\left(F_{0}, F_{1}\right)} \exp (-c d(\alpha, \beta))$ where $c=-\ln \vartheta n_{\mathfrak{G}}$.

Proof. Each term in (23) has norm at most

$$
\|\pi\|_{\mathfrak{L}(F)}^{2}\left\|R^{\prime}(\lambda)\right\|_{\mathfrak{L}\left(F_{0}, F_{1}\right)} \vartheta^{k}
$$

The number of such terms is the number of paths of length $k+2$ from $\alpha$ to $\beta$. Since each vertex in the graph $\mathfrak{G}$ is connected to at most $n_{\mathfrak{G}}$ other vertices (including itself) there at 
most $n_{\mathfrak{G}}^{k+2}$ paths emanating from $\alpha$. On the other hand, by definition there are no paths from $\alpha$ to $\beta$ of length less than $d(\alpha, \beta)$ so we can estimate the sum (23) by

$$
\begin{aligned}
\left\|\left\{(\lambda-A)^{-1}\right\}_{\alpha \beta}\right\|_{\mathfrak{L}\left(F_{0}^{(\beta)}, F_{1}^{(\alpha)}\right)} & \leq \sum_{k+2 \geq d(\alpha, \beta), k \geq 0} C\left\|R^{\prime}(\lambda)\right\|_{\mathfrak{L}\left(F_{0}, F_{1}\right)} \vartheta^{k} n_{\mathfrak{G}}^{k+2} \\
& \leq C^{\prime}\left\|R^{\prime}(\lambda)\right\|_{\mathfrak{L}\left(F_{0}, F_{1}\right)} \frac{\left(\vartheta n_{\mathfrak{G})} d(\alpha, \beta)-2\right.}{1-\vartheta n_{\mathfrak{G}}} \\
& \leq \frac{2 C^{\prime}}{\left(\vartheta n_{\mathfrak{G}}\right)^{2}}\left\|R^{\prime}(\lambda)\right\|_{\mathfrak{L}\left(F_{0}, F_{1}\right)} \exp (-c d(\alpha, \beta)) \\
& =C\left(\lambda, A^{\prime}, \vartheta n_{\mathfrak{G}}\right) \exp (-c d(\alpha, \beta))
\end{aligned}
$$

where $c=-\ln \vartheta n_{\mathfrak{G}}$, as promised. Q.E.D.

\section{EXPONENTIAL DECAY OF EIGENFUNCTIONS}

Theorem 6.1. Let $\lambda_{0}$ be an isolated eigenvalue with finite multilpicity of $A \in H o l(E)$. Assume there exists a localized resolution $A^{\prime}$ of $A$ and a smooth closed curve $\Gamma \subset \mathbb{C}$ contained in the resolvent set of $A^{\prime}$, such that $\lambda_{0}$ is the only point in the spectrum of $A$ lying inside of $\Gamma$. Keeping the same notation as in the previous section we assume that for some $\vartheta$ with $\vartheta n_{\mathfrak{G}} \leq \frac{1}{2}$ the estimate (20) holds for all $\lambda \in \Gamma$.

Then any generalized eigenfunction $v \in E_{1}$ of $A$ corresponding to the eigenvalue $\lambda_{0}$ is exponentially localized, i.e. for some $\alpha_{1} \in \mathfrak{A}$ one has

$$
\left\|(\delta v)_{\beta}\right\|_{F_{0}} \leq C \exp \left(-c d\left(\beta, \alpha_{1}\right)\right) .
$$

Proof. The hypotheses imlpy that $\Gamma$ also lies in the resolvent set of $A$.

Corresponding to any closed curve in the resolvent set of an operator $A$ one can define the spectral projection $P_{\Gamma}$ by a Dunford integral

$$
P_{\Gamma} \stackrel{\text { def }}{=} \oint_{\Gamma}(\lambda-A)^{-1} \frac{d \lambda}{2 \pi i}
$$

If $\Gamma$ encloses one and only one eigenvalue $\lambda_{0}$ of $A$ then $P_{\Gamma}$ is the projection on the generalized eigenspace corresponding to $\lambda_{0}$. The matrix elements with respect to the localized resolution $E \stackrel{\delta}{\longrightarrow} \bigoplus_{\alpha \in \mathfrak{A}} F^{(\alpha)} \stackrel{\varepsilon}{\longrightarrow} E$ are then given by

$$
\left(P_{\Gamma}\right)_{\alpha \beta} \stackrel{\text { def }}{=} \oint_{\Gamma}\left\{(\lambda-A)^{-1}\right\}_{\alpha \beta} \frac{d \lambda}{2 \pi i}
$$

By theorem 5.2 we can estimate this operator by

$$
\begin{aligned}
\left\|\left(P_{\Gamma}\right)_{\alpha \beta}\right\| & \leq C|\Gamma|\left(\vartheta n_{\mathfrak{G}}\right)^{-2} \sup _{\lambda \in \Gamma}\left\|R^{\prime}(\lambda)\right\|_{\mathfrak{L}\left(F_{0}, F_{1}\right)} \exp (-c d(\alpha, \beta)) \\
& =C\left(\Gamma, A^{\prime}, \vartheta n_{\mathfrak{G}}\right) \exp (-c d(\alpha, \beta))
\end{aligned}
$$

with $c=-\ln \vartheta n_{\mathfrak{G}}$.

Assume now that $\Gamma$ encloses one and only one eigenvalue $\lambda_{0}$ of $A$, and that $P_{\Gamma}$ has finite $\operatorname{rank} N$ (so $\lambda_{0}$ is an eigenvalue of finite multiplicity). Since the space $F_{1}^{\text {fin }}$ of $x \in F_{0}$ with finite support is dense in $F_{0}$ there are $x_{1}, \ldots, x_{N} \in F_{0}^{\text {fin }}$ such that $P_{\Gamma} x_{1}, \ldots, P_{\Gamma} x_{N}$ span 
the range of $P_{\Gamma}$. If the $x_{k}$ are contained in $F_{0}^{\left(\alpha_{1}\right)} \oplus \ldots \oplus F_{0}^{\left(\alpha_{m}\right)}$ then (26) implies that the components of $v_{k}=P_{\Gamma} x_{k}$ decay exponentially:

$$
\left\|\left(v_{k}\right)_{\beta}\right\|_{F_{1}} \leq C \sum_{i=1}^{m} e^{-c d\left(\alpha_{i}, \beta\right)} \leq C^{\prime} e^{-c d\left(\alpha_{1}, \beta\right)}
$$

(we have used the triangle inequality: $d\left(\alpha_{i}, \beta\right) \leq d\left(\alpha_{1}, \beta\right)+d\left(\alpha_{i}, \alpha_{1}\right)$.)

The same estimate applies to any generalized eigenfunction $v=C_{1} v_{1}+\ldots+C_{m} v_{m}$. Q.E.D.

\section{EXPONENTIAL DECAY IN THE EXAMPLE}

We return to the example in section 4. In this section we choose a slightly different resolution $A^{\prime}=\bigoplus A^{(\alpha)}$, namely we set all $A^{(\alpha)}$ equal to the same operator $A=$ $\sum_{|j| \leq m} a_{j}(x) D^{j}$. Thus the coefficients $a_{j}^{(\alpha)}(x)$ coincide with the coefficients $a_{j}(x)$ of $A$, and the computation (16) implies that the $\alpha \beta$-matrix element of $\delta[A, \epsilon]$ is given by

$$
(\delta[A, \epsilon])_{\alpha \beta}=\sum_{|j| \leq m} \sum_{0<k \leq j} \varphi_{\alpha ; r}(x) \frac{b_{j ; k}^{(\beta)}(x)}{r^{|k|}} \circ D^{j-k} .
$$

Since the $b_{j ; k}^{(\beta)}(x)$ and $\varphi_{\alpha ; r}(x)$ are uniformly bounded in $L_{\infty}\left(\mathbb{R}^{d}\right)$ (i.e. independently of $r>0)(\delta[A, \epsilon])_{\alpha \beta}$ is a bounded operator from $W_{p}^{m-1}\left(\mathbb{R}^{d} ; \mathbb{R}^{n}\right)$ to $L_{p}\left(\mathbb{R}^{d} ; \mathbb{R}^{n}\right)$. Assuming that $r \gg 1$ the terms with the largest norm in (28) are those for which $|k|=1$, so that we get the following estimate for the operator norm of $(\delta[A, \epsilon])_{\alpha \beta}$

$$
\left\|(\delta[A, \epsilon])_{\alpha \beta}\right\|_{\mathfrak{L}\left(W_{p}^{m-1}\left(\mathbb{R}^{d} ; \mathbb{R}^{n}\right) ; L_{p}\left(\mathbb{R}^{d} ; \mathbb{R}^{n}\right)\right)} \leq \frac{C}{r} \quad \text { for } r \geq 1
$$

For small $r$ the terms with $|k|=|j|=m$ dominate, and one gets

$$
\left\|(\delta[A, \epsilon])_{\alpha \beta}\right\|_{\mathfrak{L}\left(W_{p}^{m-1}\left(\mathbb{R}^{d} ; \mathbb{R}^{n}\right) ; L_{p}\left(\mathbb{R}^{d} ; \mathbb{R}^{n}\right)\right)} \leq \frac{C}{r^{m}} \text { for } 0<r \leq 1
$$

Composing $(\delta[A, \epsilon])_{\alpha \beta}$ from the left or right with $R^{\prime}(\lambda)$ and keeping in mind that the action of $R^{\prime}(\lambda)$ on $F^{(\alpha)}$ or $F^{(\beta)}$ is the same as that of $R(\lambda)=(\lambda-A)^{-1}$, we find that (20) is satisfied provided one takes $\vartheta=\frac{C}{r}\|R(\lambda)\|_{\mathcal{L}\left(L_{p}\left(\mathbb{R}^{d} ; \mathbb{R}^{n}\right) ; W_{p}^{m}\left(\mathbb{R}^{d} ; \mathbb{R}^{n}\right)\right)}$.

Theorem 7.1. Let $\lambda_{0}$ be an isolated eigenvalue with finite multiplicity of the operator $A$ of section 4. Then any generalized eigenfunction $v(x) \in W_{p}^{m}\left(\mathbb{R}^{d} ; \mathbb{R}^{n}\right)$ of $A$ is exponentially decaying at $\infty$, i.e. for certain constants $c, C<\infty$ one has

$$
|v(x)| \leq C e^{-c|x|}
$$

Proof. Choose a closed contour $\Gamma$ around $\lambda_{0}$, e.g. one could let $\Gamma$ be a sufficiently small circle centered at $\lambda_{0}$. Then the resolvents $R(\lambda)$ are uniformly bounded from $L_{p}$ to $W_{p}^{m}$ for all $\lambda \in \Gamma$, and by (29) the condition (20) holds with $\vartheta=\frac{C}{r}$, where $\sup _{\lambda \in \Gamma}\|R(\lambda)\|$ is incorporated into the constant $C$. If one chooses $r$ large enough, then theorem 6.1 applies, and gives us exponential decay for $\left\|\varphi_{\alpha ; r}(x) v(x)\right\|_{L_{p}}$. Since the $v(x)$ are generalized eigenfunctions of an elliptic operator with continuous coefficients one can easily translate the $L_{p}$ estimates into pointwise estimates and thus obtain the pointwise exponential decay claimed in (31).

Q.E.D. 
So far we have not really exploited the fact that $(\delta[A, \epsilon])_{\alpha \beta}$ is a differential operator of order less than $m$. Choose an $\omega \in \mathbb{R}_{+}$such that $\lambda-A$ is invertible with $\|R(\lambda)\|_{\mathfrak{L}\left(L_{p} ; W_{p}^{m}\right)} \leq$ $C$ for all $\lambda \in \mathbb{C}$ with $\Re \lambda>\omega$. Then we also have $\|R(\lambda)\|_{\mathfrak{L}\left(L_{p} ; L_{p}\right)} \leq \frac{C}{|\lambda|}$ for all $\lambda$ with $\Re \lambda \geq \omega$. Interpolation between $W_{p}^{m}$ and $L_{p}$ then implies that

$$
\left\|D^{j-k} \circ R(\lambda)\right\|_{\mathfrak{L}\left(L_{p}, L_{p}\right)} \leq C|\lambda|^{-1+\frac{|j|-|k|}{m}} .
$$

Combine this with (28) and you get the following estimate for the $L_{p} \rightarrow L_{p}$ norm of the operator $(\delta[A, \epsilon] R(\lambda))_{\alpha \beta}$

$$
\begin{aligned}
\left\|\left(\delta[A, \epsilon] R^{\prime}(\lambda)\right)_{\alpha \beta}\right\|_{\mathfrak{L}\left(L_{p}, L_{p}\right)} & \leq \sum_{|j| \leq m} \sum_{0<k \leq j}\left\|\varphi_{\alpha ; r}(x) \frac{b_{j ; k}^{(\beta)}(x)}{r^{|k|}} \circ D^{j-k} \circ R(\lambda)\right\| \\
& \leq C \sum_{|j| \leq m} \sum_{0<k \leq j} r^{-|k|}|\lambda|^{-1+\frac{|j|-|k|}{m}} \\
& \leq C \sum_{|j| \leq m} \sum_{0<k \leq j}\left(r|\lambda|^{1 / m}\right)^{-|k|} \\
& \leq \frac{C}{r|\lambda|^{1 / m}}
\end{aligned}
$$

assuming that $r|\lambda|^{1 / m} \geq 1$. Since $\delta[A, \epsilon] R^{\prime}(\lambda)$ is dominated by the projection $\pi_{F}$ we also get

$$
\left\|\delta[A, \epsilon] R^{\prime}(\lambda)\right\|_{\mathfrak{L}\left(F_{0}\right)} \leq \frac{n_{\mathfrak{G}} C}{r|\lambda|^{1 / m}}
$$

Hence if $r|\lambda|^{1 / m}$ is sufficiently large and if $\Re \lambda \geq \omega$, then the resolvent $R(\lambda)$ is given by (1) and its matrix elements are given by (23). With $r=\frac{2 C}{n_{\mathfrak{G}}|\lambda|^{1 / m}}$ we get $\vartheta n_{\mathfrak{G}}=\frac{1}{2}$ so that theorem 5.2 implies

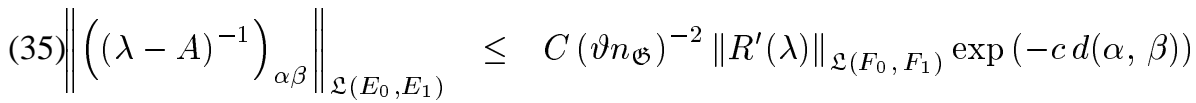

$$
\begin{aligned}
& \leq \quad C^{\prime} \exp (-c d(\alpha, \beta)) .
\end{aligned}
$$

Refering to the interpretation of the matrix element given in (14) we see that the size of $(\lambda-A)^{-1} f$ in $O_{\alpha ; r}$ where $f$ is a function which is localized in $O_{\beta ; r}$ is at most $C^{\prime} e^{-c d(\alpha, \beta)}\|f\|$. The proper measurement of the exponential $e^{-c d(\alpha, \beta)}$ is in terms of the distance between the sets $O_{\alpha ; r}$ and $O_{\beta ; r}$, rather than between the indices $\alpha, \beta \in \mathfrak{A}=\mathbb{Z}^{d}$. The distance between $O_{\alpha ; r}$ and $O_{\beta ; r}$ is proportional to $r|\alpha-\beta|$, and the graph distance $d(\alpha, \beta)$ is also roughly proportional to $|\alpha-\beta|$. Hence we get the following estimate

$$
\left\|\left((\lambda-A)^{-1}\right)_{\alpha \beta}\right\|_{\mathfrak{L}\left(E_{0}, E_{1}\right)} \leq C^{\prime} \exp \left(-c|\lambda|^{1 / m}\left|x_{\alpha}-x_{\beta}\right|\right)
$$

for all $\lambda$ with $\Re \lambda \geq \omega$. (We have written $x_{\alpha}=r \alpha$ for the centre of $O_{\alpha ; r}$.) Using elliptic interior estimates for the equation $\lambda u-A u=f$ one can show that the resolvent $(\lambda-A)^{-1}$ has an integral kernel $K(\lambda ; x, y)$ which is bounded by

$$
|K(\lambda ; x, y)| \leq C^{\prime} \exp \left(-c|\lambda|^{1 / m}|x-y|\right) \quad \text { for }|x-y| \geq C|\lambda|^{-1 / m}
$$




\section{Application to "Fredholm solutions" of Nonlinear Elliptic Systems}

When studying solutions of systems of nonlinear elliptic PDE from the point of view of bifurcation theory (as in $[7,8]$ ) it is of interest to consider the linearized equation. Theorems from bifurcation theory will in general only be applicable if the linearized operator is a Fredholm operator. The following theorem shows that a slightly stronger condition will not hold for most bounded entire solutions of elliptic PDEs.

Theorem 8.1. Let $u \in B U C^{2}\left(\mathbb{R}^{d} ; \mathbb{R}^{n}\right)$ be a solution of the nonlinear PDE

$$
F\left(u, D u, D^{2} u, \ldots, D^{m} u\right)=0
$$

where $F$ is a $C^{1}$ function of its arguments, and assume that the linearized operator

$$
A \stackrel{\text { def }}{=} \sum_{|j| \leq m} a_{j}(x) D^{j}, \quad a_{j}(x) \stackrel{\text { def }}{=} \frac{\partial F}{\partial\left(D^{j} u\right)}\left(u(x), \ldots, D^{m} u(x)\right)
$$

is uniformly elliptic. If $\lambda=0$ does not belong to the essential spectrum of the operator $A: W_{p}^{m}\left(\mathbb{R}^{d} ; \mathbb{R}^{n}\right) \rightarrow L_{p}\left(\mathbb{R}^{d} ; \mathbb{R}^{n}\right)$ then $\lim _{|x| \rightarrow \infty} u(x)$ exists. (In case $d=1$ the limits at $+\infty$ and $-\infty$ may be different.)

Proof. By differentiating the equation (39) with respect to $x_{k}$, one finds that $\partial_{k} u$ is an eigenfunction of $A$ with eigenvalue 0 . Hence by theorem 7.1 all derivatives $\partial_{k} u$ decay exponentially as $|x| \rightarrow \infty$. By integrating the gradient along circles centered the origin one sees that the oscillation of $u(x)$ on the sphere of radius $r$ decays exponentially as $r \rightarrow \infty$ (at least if $d \geq 2$, otherwise such circles do not exist.) By integrating along rays one sees that $u(x)$ actually tends to a constant. Q.E.D.

\section{REFERENCES}

[1] H.Amann, Quasilinear evolution equations and parabolic systems., TAMS 293 (1986), 191 - 227.

[2] H.Amann, On abstract parabolic fundamental solutions, J. Math. Soc. Japan, 39(1987).

[3] H.Amann, Quasilinear parabolic systems under nonlinear boundary conditions., Arch. Rat. Mech. Anal. 92 (1986), 153 - 192.

[4] H. Amann, Linear and Quasilinear Parabolic Problems, Vol II, Birkhauser, to appear.

[5] H. Amann, M. Hieber, G. Simonett: Bounded $H^{\infty}$-calculus for elliptic operators, Differential and Integral Equations 7 (1994), 613-653.

[6] Angenent, S. B., Nonlinear Analytic Semiflows, Proceedings of the Royal Society in Edinburgh 115A(1990) pp.91-107.

[7] Angenent, S. B., The shadowing lemma for elliptic PDE, pp.7-22 in "Dynamics of Infinite dimensional dynamical systems," NATO/ASI series F, vol.37, Springer Verlag.

[8] Angenent, S. B., A variational interpretation of Melnikov's function and exponentially small separatrix splitting, pp.5-35 in "Symplectic Geometry," London Mathematical Society Lecture Note Series vol.192, Cambridge University Press (1993)

[9] Bergh, J. AND J.LÖFström, Interpolation spaces, an introduction, Springer Verlag, Berlin, 1976.

[10] Butzer, P.L. AND H. BERENS , Semigroups of operators and approximation, Springer Verlag, Berlin, 1967.

[11] Clément, Ph., H.J.A.M.Heijmans, ET. Al., One Parameter Semigroups, CWI Monographs \#5, North Holland, Amsterdam, 1987.

[12] DAPRATO, G. AND P. GRISVARD, Équations d'évolutions abstraites nonlinéair de type parabolique, Annali di Mat. Pura ed Appl., 120 (1979), 329 - 396.

[13] LunARdi, A., Quasilinear parabolic equations, Math. Annalen 267 (1984), 395 - 415.

[14] PAZY, A, Semigroups of linear operators and applications to partial differential equations, Springer Verlag, New York, 1983.

[15] Triebel, H., Interpolation theory, function spaces, differential operators, North Holland, Amsterdam, 1978 